\title{
Educar en la escuela infantil del siglo XXI: diálogo, inclusión y tecnología
}

\author{
Educating in the infant school of the $21^{\text {st }}$ century: \\ dialogue, inclusion and technology
}

Isabel M. Gallardo-Fernández

Departamento de Didáctica y Organización Escolar, Universidad de Valencia, España

isabel.gallardo@uv.es

Héctor Saiz-Fernández

Departamento de Didáctica y Organización Escolar, Universidad de Valencia, España saizfer@alumni.uv.es

\section{Miriam Elizabeth Aguasanta Regalado}

Departamento de Didáctica y Organización Escolar, Universidad de Valencia, España mieare@alumni.uv.es

\section{Mónica López Iglesias}

Departamento de Didáctica y Organización Escolar, Universidad de Valencia, España loimo@alumni.uv.es

\section{RESUMEN}

Los objetivos de la educación del siglo XXI tratan de garantizar una educación inclusiva, de calidad y equitativa, que propicie oportunidades educativas para todos los infantes. Cada vez más, los menores son expuestos a un mayor tiempo de consumo digital. Ante el aumento del número de pantallas en el hogar, así como de recursos digitales nos planteamos: ¿cuáles son las características de la infancia actual?, ¿qué lugar ocupan las tecnologías en la vida del niño?, ¿y en la del docente de infantil? En este artículo se realiza una revisión bibliográfica que trata de responder a los interrogantes planteados. El objetivo es elaborar el estado del arte en relación al desarrollo e implementación de lo digital en Educación Infantil. A diario, observamos cómo los niños manipulan la tablet antes de aprender a hablar o escribir y que apenas saben lo que es jugar en el mundo real. Estamos ante un tema complejo por lo que es preciso garantizar el pleno desarrollo del niño y seguir indagando en los avances de la investigación sobre infancia, inclusión y tecnología. Los resultados evidencian que las tecnologías son una oportunidad para lograr cambios profundos que contribuyan a mejorar los procesos de enseñanza aprendizaje desde nuevos planteamientos pedagógicos y curriculares.

PALABRAS CLAVE Educación Preescolar, Tecnología Educativa, Rol del Estudiante, Rol del Docente.

\section{ABSTRACT}

The objectives of education for the 21st century seek to guarantee an inclusive, quality and equitable process that fosters educational opportunities for all infants. Increasingly, minors are exposed for a longer time of digital consumption. Because of the increase in the number of screens in the home, as well as digital resources, we ask ourselves: What are the characteristics of today's childhood? What place do technologies occupy in children and preschool teachers' lives? This article makes 
a bibliographic review that tries to answer these questions. The main objective is to elaborate the state of the art in relation to the development and implementation of digital technology in childhood education. Every day we observe how children manipulate the tablet before learning to speak or write and that they hardly know what it is to play in the real world. We are facing a complex issue, so it is necessary to guarantee the full development of the child and continue investigating the advances in research on childhood, inclusion and technology. The results show that technologies are an opportunity to achieve profound changes that contribute to improving the teaching-learning processes from new pedagogical and curricular approaches.

KEYWORDS Preschool Education, Educational Technology, Student Role, Teacher Role.

\section{INTRODUCCIÓN}

“La educación más alta es la que no se limita a inculcarnos conocimientos, sino que pone nuestra vida en armonía con todo lo existente" (Tagore, 1917, p.60).

El sistema educativo español ha evolucionado notablemente en los últimos veinte años. La relevancia del proceso educativo como garantía para el desarrollo integral del sujeto en un contexto cívico y de ciudadanía, se ha venido ensalzando de forma escalonada. Si bien, el sistema educativo también ha experimentado diversas crisis que han golpeado sus cimientos. Pese a todo, el horizonte de la inclusión social y educativa parece permanecer y enraizar en el marco legislativo y curricular.

Hoy en día, los planteamientos normativos sobre educación oscilan entre la calidad y la equidad (Murillo, \& Martínez, 2018; Saiz, 2019). Estamos ante dos enfoques hacia los que se trata de avanzar de forma coherente e inclusiva (Fernández, 2013). Asumimos que existe un sólido consenso mundial sobre la necesidad y la importancia de educar para los derechos humanos, la tolerancia, la convivencia y la vida en democracia. Y, también, sobre la responsabilidad de los estados en implementar tal educación para todos sus ciudadanos. Este consenso quedó plasmado ya en la Declaración Universal de los Derechos Humanos de 1948. De allí en adelante se ha ido ampliando y profundizando en otros instrumentos de derechos humanos (Declaración de los Derechos del Niño de 1959) así como declaraciones de organismos internacionales. Especialmente las Naciones Unidas (UNESCO, 2009) se han comprometido a fortalecer la tolerancia mediante el fomento de la comprensión mutua entre las culturas y los pueblos. Este imperativo está en la base de la Carta de las Naciones Unidas y de la Declaración Universal de los Derechos Humanos y precisamente, en los tiempos tan inciertos que estamos viviendo en plena pandemia, hemos de remar a favor del diálogo, la tolerancia y el cuidado de la vida humana. La tolerancia reconoce los derechos humanos universales y la diversidad de los pueblos. Morin (2020) lo expresa así: “(...) hagamos todo lo que esté en nuestras manos para regenerar la política, proteger el planeta y humanizar la sociedad. Es hora de cambiar de vía” (p. 21). Ciertamente que el autor plantea un cambio de paradigma. pese a ser un proceso complejo. Sin embargo, considera que hemos de caminar hacia sistemas sostenibles que prioricen el cuidado de las personas frente a los criterios neoliberales del presente.

Nuestra formación personal y profesional en el mundo de la educación nos sitúa en la perspectiva de investigar y profundizar sobre los derechos de la infancia, especialmente por su protección, con horizontes 
a corto plazo, tal como se plantea en la Agenda 2030 a través de los Objetivos de Desarrollo Sostenible (UNESCO, 2015). A este respecto, trabajamos e incluimos los contenidos derivados de estas consideraciones como parte fundamental de la práctica docente, estando estos contenidos curriculares en estrecha relación con los fines culturales y sociales de la comunidad (Gimeno, 2013).

Hemos de destacar también el Plan Estratégico de UNICEF para 2018-2021, que se formuló con el objeto de impulsar la protección a la infancia en un mundo cambiante. "Por primera vez, el Plan Estratégico traza un rumbo para los próximos cuatro años, con miras a la consecución de los Objetivos de Desarrollo Sostenible en 2030 y la materialización de un mundo mejor para cada niño donde nadie quede excluido" (UNICEF, 2018 , p. 6). En su articulado, conceden especial valor a la tecnología como herramienta de inclusión y favorecedora del aprendizaje en aquellos contextos más vulnerables.

En el marco del Informe La educación encierra un tesoro, de la Comisión Internacional sobre la Educación para el Siglo XXI (Delors, 1996), el aprendizaje por competencias constituye la base metodológica del actual proceso de aprendizaje. Entre las siete competencias clave descritas en la normativa educativa, destaca en relación con este artículo, la competencia digital que supone un enfoque de trabajo globalizado, el cual necesariamente involucra a la comunidad educativa y al resto de la población en base al principio de esfuerzo compartido.

La implementación e incorporación de las Tecnologías de la Información y la Comunicación (TIC) en los centros educativos no siempre ha seguido un patrón progresivo. En algunos casos, la tecnología ha irrumpido en las aulas haciendo que sea preciso reflexionar sobre su valor pedagógico y analizar las prácticas docentes que las TIC facilitan y promueven en el contexto escolar, así como los cambios en el rol docente en el contexto de la sociedad digital (García, \& Gutiérrez, 2020).

Respecto al contexto educativo, la aplicación de las TIC da lugar a las Tecnologías del Aprendizaje y el Conocimiento (TAC), siendo estas cada vez más precisas y específicas en el panorama educativo formal. Su evolución y actualización constante se manifiesta incluso en su nomenclatura, la cual se adapta para poder acoger los avances y cambios. Actualmente, se utiliza también el concepto de Tecnologías de la Relación, la Información y la Comunicación (TRIC), con el objetivo de acoger la presencia y relevancia de las redes sociales como forma de relación. Con el fin de agilizar este texto, empleamos TIC como forma de englobar el conjunto de tecnologías que abarca la realidad.

En el contexto actual de educación líquida, caracterizado por la volatilidad, la incertidumbre y la inseguridad (Bauman, 2020), donde las tecnologías ya no son ninguna novedad y el flujo de información es colosal y caótico, resulta preciso adquirir perspectiva sobre el tema que nos ocupa. Por ello, en este artículo tratamos de localizar y analizar las investigaciones previas que hasta este momento se han realizado sobre el desarrollo infantil desde un enfoque inclusivo y en relación con las tecnologías. De este modo, lograremos concretar el estado del arte del tema que nos ocupa, lo que fundamentará el objeto de la propia investigación y nos permitirá posicionarnos desde una perspectiva más amplia (Vargas, \& Calvo, 1987).

\section{DISEÑO Y METODOLOGÍA}

En un contexto como el actual, donde las TIC han potenciado un desarrollo hacia un horizonte más tecnológico, pero no siempre con la adecuada tarea de reflexión sobre su sentido y contribuciones a largo plazo, es necesario tomar distancia y analizar su avance (González, 2007). El propósito de esta investigación es 
elaborar el estado del arte sobre la evolución del contexto de la Educación Infantil en relación al desarrollo e implementación de lo digital en esta etapa, así como a las características de la infancia actual y su relación con los procesos y mecanismos de inclusión educativa.

En este artículo, nuestro objetivo se centra en presentar una imagen representativa de lo que supone educar en la escuela infantil del siglo XXI a partir de tres categorías de análisis: las características de la infancia, la relación de la infancia con las TIC y la relación de las maestras/os de infantil con las tecnologías.

En cuanto a los procedimientos utilizados para la realización del estado del arte, nos apoyamos en los cuatro pasos que Molina (2005) sugiere para su realización.

En primer lugar, concretamos los criterios que han definido la búsqueda del material documental. En este caso, la búsqueda se realiza entre bases de datos, tesis doctorales, repositorios universitarios, catálogos de bibliotecas y revistas especializadas. Específicamente, la búsqueda se realizó a través de las siguientes plataformas: ERIC, WorldCat, Google Scholar, Dialnet, TESEO, SCOPUS, Red de Revistas Científicas de América Latina y el Caribe, España y Portugal, REDIB y catálogo virtual TROBES.

Para realizar la búsqueda se utilizaron las siguientes palabras clave combinadas de diferentes modos, en español e inglés: educación, infantil, tecnología, TIC, pantalla, inclusión, inclusivo. La búsqueda se concreta en documentos publicados desde 2010 a 2021.

En segundo lugar, con el fin de sistematizar los contenidos resultantes de la búsqueda, organizamos los resultados en función de tres categorías que responden a las cuestiones que nos planteamos al inicio de este artículo: a) características de la infancia actual; b) relación de las TIC y la infancia y c) relación entre las tecnologías y los docentes de Educación Infantil.

En tercer lugar, se concreta la información de los documentos encontrados en función de cada una de las categorías de análisis. Dicha información se analiza y se expone de forma breve y sintética con el fin de referenciar la información más relevante.

Por último, en cuarto lugar, se desarrollan una serie de conclusiones sobre los estudios analizados para intentar encontrar patrones relevantes que nos favorezcan para adquirir esa perspectiva amplia que nos planteamos como objetivo.

El proceso metodológico para la elaboración del estado del arte queda representado en la Figura 1.

Figura 1. Proceso metodológico del estado del arte según Molina (2005). Fuente: elaboración propia.

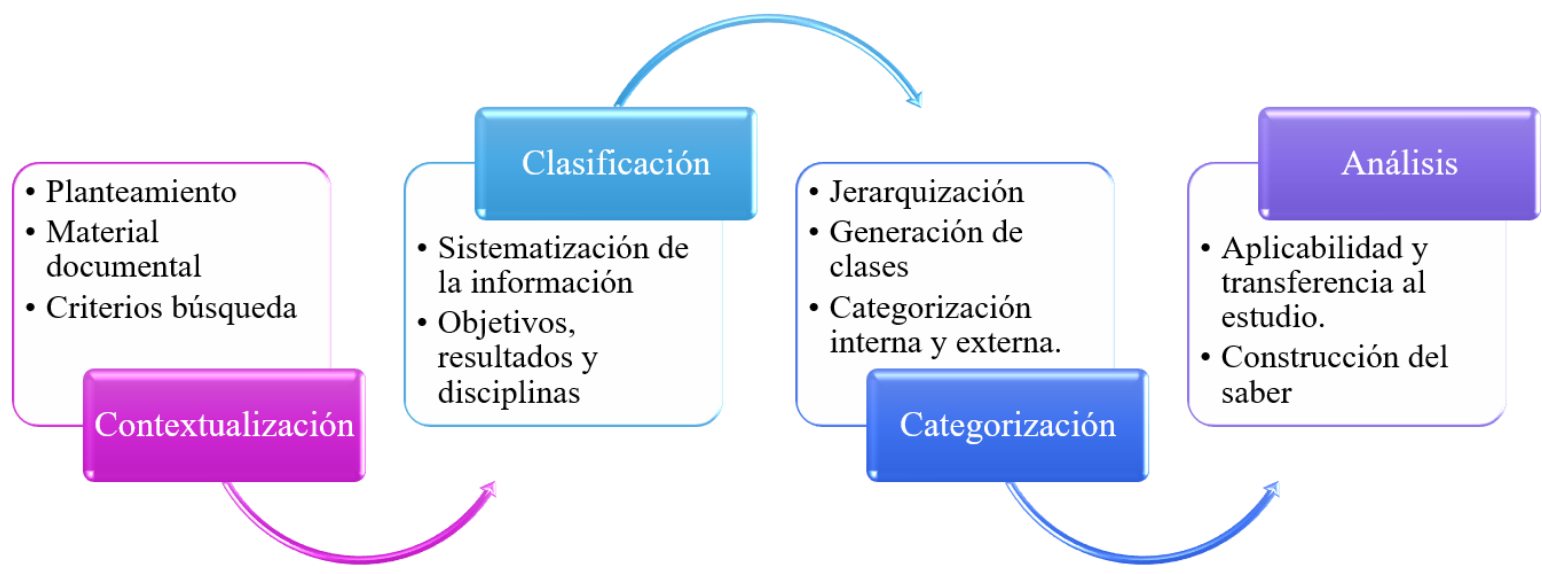




\section{RESULTADOS}

Los resultados se organizan en tres núcleos de información a partir de las categorías de análisis planteadas: características de la infancia, relación de las TIC y la infancia y relación entre las TIC y los maestros y maestras de Educación Infantil. Respecto a la búsqueda realizada, cabe señalar que la dimensión de las TIC y los dispositivos digitales constituyen un objetivo de análisis en el total de estudios revisados. Este hecho es generalizable al conjunto de investigaciones sobre educación, y específicamente sobre Educación Infantil. Destaca así la relevancia que las TIC han adquirido en el panorama educativo y su presencia como parte fundamental de las investigaciones educativas actuales. En relación a los resultados obtenidos tras la búsqueda, existen investigaciones que se centran en experiencias concretas de aula, en el marco de un área o de metodologías específicas (Figura 2). Sin embargo, no son tan habituales los trabajos que plantean un análisis de las repercusiones del uso de las TIC en el contexto educativo a corto, medio o largo plazo. En este sentido, no se han encontrado resultados significativos que se centren en un planteamiento metaanalítico de la cuestión.

Los resultados seleccionados tras la búsqueda, son una muestra entre el conjunto de resultados obtenidos. Los criterios de selección se basan en la significatividad del propio estudio para con este trabajo, siendo seleccionadas las investigaciones tras su revisión y comparación con los objetivos que nos planteamos.

\subsection{Características de la infancia}

En relación con la primera categoría, la infancia, suele ser representada como una etapa de grandes cambios a nivel fisiológico y psicológico. Tras realizar la búsqueda, se comprueba como, por un lado, existe una aproximación predominante hacia la infancia desde un enfoque clínico diagnóstico, que se centra en reportar características propias de los diferentes momentos de crecimiento en relación con el desarrollo psicoevolutivo de los infantes. Desde esta aproximación, estudios como los de Correa et al. (2015), Pombo (2019) y Ribeiro (2019) ofrecen una visión clínica de los conflictos que pueden suceder a lo largo de la infancia, así como estrategias de intervención para determinados trastornos asociados. Asimismo, estudios como el de Cano-Díaz et al. (2015) evidencian la falta de investigaciones que se centren en la caracterización de la infancia, objeto de nuestro interés. Por ello, nos apoyamos en el conjunto de evidencias surgidas en el proceso de búsqueda y análisis de los documentos, para aportar una imagen relevante sobre la infancia actual.

Sabido es que existen investigaciones que desarrollan una mirada pedagógica de la infancia, entendiendo que esta etapa está inmersa en un proceso educacional, como una acción humana, intencional que está orientada al perfeccionamiento de las personas y que se desarrolla en una estructura definida. Un ejemplo de investigación que representa este enfoque es el de Zabalza (2018), que analiza los dos ejes de desarrollo sobre los que se han construido las pedagogías de la infancia: la Educación Infantil como clave en el proceso de desarrollo equilibrado en los niños y niñas, y el dilema entre cuidado y educación (que enfrenta dos concepciones del ser humano como aprendiz pasivo o activo).

Desde este enfoque pedagógico, existen investigaciones como la de González (2019) y Grindheim (2017) que parten de la concepción de que ser niño o niña es una forma de habitar el mundo, no definida únicamente por el periodo evolutivo. A este respecto, las investigaciones evidencian como desde un enfoque inclusivo las ciudades, así como sus habitantes, constituyen un factor que limita o favorece ese despliegue a la 
hora de habitar el mundo como niño o niña. En el caso de Grindheim (2017), realiza una crítica de las formas en que la infancia participa en la vida ciudadana y propone el juego, la actividad de mayor importancia en la infancia, como el medio para participar en ella.

En relación al estado de la infancia respecto a criterios de exclusión social, en España "el 31\% de la población infantil, 2.610.370 millones de niños y niñas, están en riesgo de pobreza o exclusión social” según la organización Save the Children (2017, p. 16). Además, una de las características de la exclusión en la infancia, es que perdura entre generaciones. Tal como argumentan Marcos y Ubrich (2017) "en sociedades más desiguales, la igualdad de oportunidades está más condicionada por la herencia de renta y capital social y cultural que reciben los niños y niñas de sus padres y madres" (p. 14). A este respecto, la investigación de Quintana (2019) evidencia como en los últimos años, el bienestar de las familias, especialmente de la infancia, ha pasado a ser cada vez más un asunto de interés público.

Para concluir este apartado, hacemos referencia a publicaciones que se centran en aspectos específicos de la infancia, del contexto educativo y social, que adquieren especial significado en relación con situaciones que surgen a raíz del desarrollo social y comunitario actual. La investigación de Palacio et al. (2018) concluye exponiendo cómo el estrés escolar en la etapa de la infancia es hoy en día un tema destacado debido a su crecimiento, sobre el que es prioritario intervenir para asegurar el bienestar de los y las menores. Del análisis realizado, se constata que existen numerosas investigaciones que se centran en revisar aspectos como el estrés, desde la parte neurocientífica, dando lugar a un movimiento relevante sobre la aplicación de esta ciencia a la educación. Nos estamos refiriendo a la Neuroeducación que se centra en aspectos tan destacados como la educación emocional, el pensamiento creativo, así como en las funciones básicas del cerebro en relación con el aprendizaje de los niños y niñas (Dubinsky et al, 2019; Espino-Díaz, 2020; Perrin, 2019).

\subsection{Relación de las TIC y la infancia}

La segunda categoría identifica que las TIC suponen, en la actualidad, una dimensión relevante a considerar cuando se investiga sobre el proceso educativo y el desarrollo de la infancia. La búsqueda bibliográfica realizada secunda dicha afirmación, tratándose de un tema que aborda directa o indirectamente los planteamientos actuales de investigación sobre Educación Infantil. Tal como recogen Konca y Koksalan (2017), los niños y niñas viven en un ambiente donde abunda la tecnología. De su estudio se concluye que, los infantes de 4 a 6 años, consumen diariamente una media de 115 minutos de programación televisiva y 28 en el uso de dispositivos. Además, su estudio evidencia que los niños y niñas poseen las competencias básicas para el uso fluido e interactivo con las TIC. Camargo y Orozco (2013) secundan esta realidad afirmando, además, que los niños y niñas disponen de un alto grado de dispositivos personales, a su alcance, cuya prevalencia aumenta con la edad y el género masculino.

En el estudio realizado por la European Commission (2015) titulado Young children (0-8) and digital technology: a qualitative exploratory study across seven countries, se muestra que el dispositivo preferido por los menores son las tabletas, debido a la multifuncionalidad que ofrecen. En este caso, el uso más generalizado es para el juego y visionado de dibujos animados, así como también la creación a partir de su uso (dibujos, fotografía, video, entre otros).

Respecto a los resultados que arroja nuestra búsqueda, en primer lugar, hacemos referencia a la investigación de Lindahl y Folkesson (2012), quienes argumentan el conflicto que surge de la implementación de las TIC en el contexto de la Educación Infantil en relación al choque que se produce entre los planteamientos mediados 
por tecnología y la prescripción curricular. Sus resultados muestran dos posiciones encontradas: por un lado, aquellos maestros y maestras que acogen los planteamientos con tecnologías, por otro lado, los docentes que se muestran reacios y rechazan las nuevas herramientas tecnológicas. Los argumentos a favor de las tecnologías se relacionan con la concepción del niño/a como un ciudadano activo y competente en el área tecnológica. Por el contrario, los argumentos en contra evidencian el necesario control del profesional sobre el uso de lo tecnológico que contrasta con la necesidad de potenciar la autonomía del infante en edades tempranas.

Esta dualidad entre planteamientos es algo que viene siendo discutido entre profesionales de la investigación educativa. Mientras que autores como Díaz y Aladro (2016), Muñoz (2014) y Newman (2018), consideran las TIC como una amenaza para el aprendizaje lúdico y el desarrollo de los infantes, otros como Cánovas (2014) y Marqués (2014) se posicionan a favor del uso de las TIC como una poderosa herramienta para el aprendizaje. Superando esta visión dualizada, se plantean estudios como el de Bentley (2018) que analiza profundamente la transformación que supone el uso de tecnologías a nivel del colectivo infantil.

En este marco de posicionamientos definidos, encontramos el estudio de Kerckaert et al. (2015) que distinguen entre dos tipos de tecnologías empleadas en Educación Infantil a partir de los resultados obtenidos. Por un lado, señalan el uso de las TIC para el propio aprendizaje en competencias tecnológicas y actitudes relacionadas. Por otro lado, definen un uso de las TIC empleadas para desarrollar contenidos y atender necesidades individuales. En este sentido, relaciona ambos tipos de uso directamente con la formación inicial y la actitud del docente de infantil hacia la tecnología. Interesante matiz que retomaremos al reflexionar sobre la relación entre las TIC y los maestros y maestras de Educación Infantil.

Profundizando en la relación de las TIC en el proceso de enseñanza aprendizaje y proceso psicoevolutivo de los infantes, Leo et al. (2018) exponen la necesaria formación y educación tecnológica y audiovisual que capacite a los niños y niñas para desarrollarse de forma crítica y reflexiva. Para ello, consideran que todo el entorno educativo ha de cambiar y readaptarse tras una adecuada dotación de servicios, dinamización de espacios y formación del docente.

Como podemos constatar, son variadas las investigaciones que relacionan las tecnologías con necesidades específicas de aprendizaje derivadas, entre otras, de condiciones de diversidad funcional, dificultades de aprendizaje y condiciones específicas de salud/situación social. Son en estas investigaciones donde más referencia se hace a las TIC como medio y herramientas para la inclusión educativa e inclusión social. En este sentido, destacan las investigaciones de Andersen y Sorensen (2017), García-Perales y Almeida (2019), Marcino (2018), Mohaned (2018), Mølster (2018) y Wamuyu (2017). En el estudio que la Fundación PACER (2014) realiza, se definen cinco dimensiones en las que los niños y niñas con diversidad funcional son beneficiados por el uso de las tecnologías de apoyo en el contexto de Educación Infantil. Las cinco dimensiones se concretan en: habilidades sociales, habilidades comunicativas, capacidad de atención, habilidades motrices finas y gruesas, así como autoconfianza e independencia.

En relación al contexto educomunicativo, investigaciones como las de Cantillo (2015) y Navarro (2014) analizan el consumo y transmisión de contenidos audiovisuales que son consumidos por niños y niñas, así como su relación con la configuración de la cultura y los valores durante la infancia.

En relación al conjunto de investigaciones existentes realizadas sobre aspectos específicos relacionados con las TIC y el contexto de infantil, señalamos como representativas las de Espigares-Gámez, et al. (2020), Gómez (2016), Norehan et al. (2019), Pinto y Osório (2019), Ramada (2017), Sánchez et al. (2018) y Siraj-Blatchford y Romero (2017). La Figura 2 sintetiza los temas en los que se centran. 
Figura 2. Relación de los temas e investigaciones específicas en relación a las TIC y la Educación Infantil. Fuente: elaboración propia.

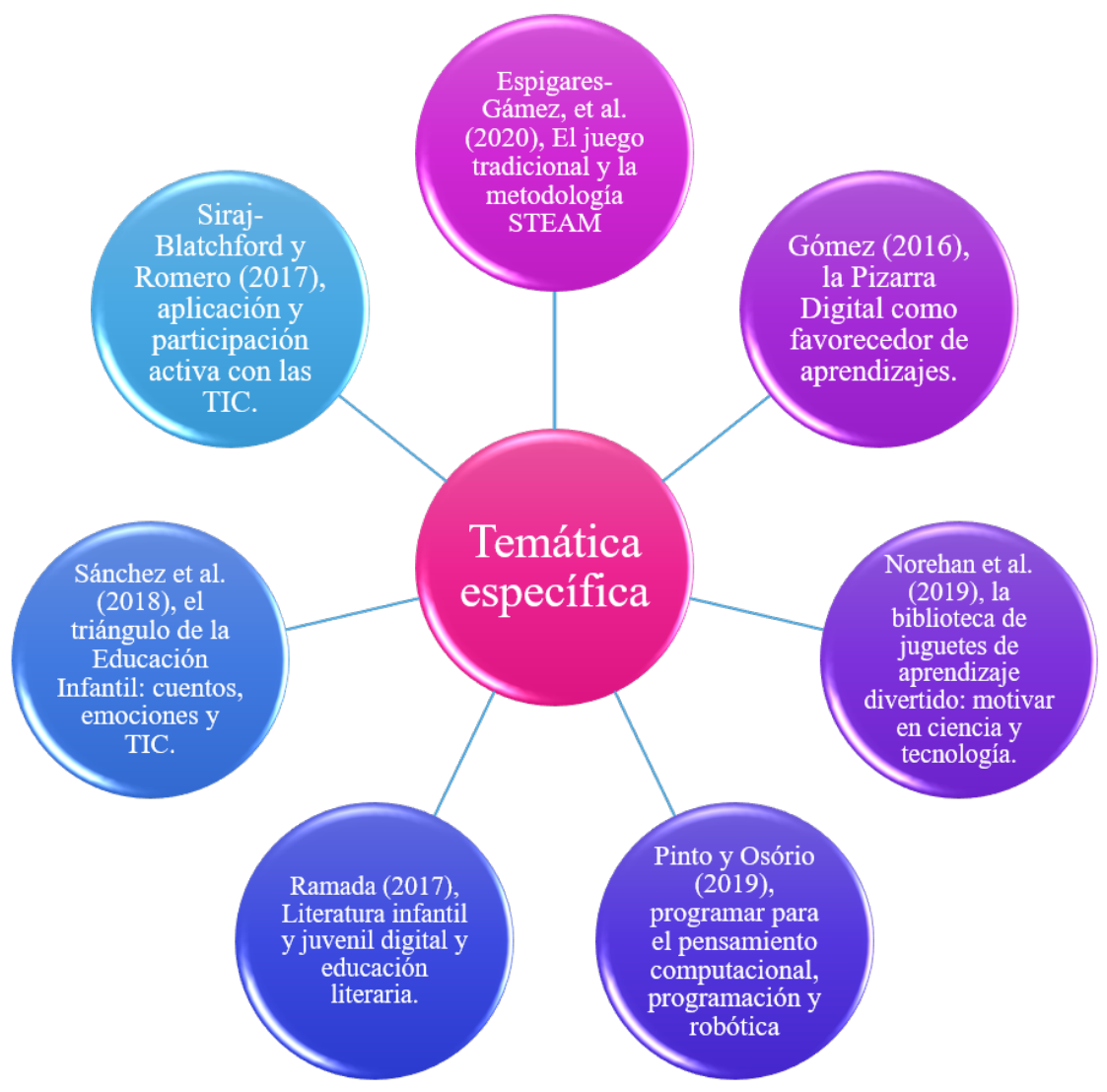

\subsection{Relación entre las TIC y los maestros y maestras de Educación Infantil}

Las tecnologías suponen un amplio abanico de oportunidades para los docentes y una forma de enriquecer su práctica. Además, se convierten en una opción de formación a través del feedback de otros docentes mediante comentarios, opiniones o recursos compartidos. Al mismo tiempo, esto tiene un efecto positivo directo sobre los recursos que se ofrecen en el aula, ya que a través de las tecnologías los alumnos tienen la posibilidad de aproximarse a nuevas realidades y culturas sin necesidad de cambiar de espacio, estimular su imaginación o potenciar su creatividad. (Masoumi, 2015).

Esta amplia oferta de recursos y herramientas implica un adecuado dominio de las TIC por parte del profesorado, por lo que es necesario dedicar un espacio a la formación continua de los docentes en este ámbito dentro del marco escolar. Según Konca et al. (2016), para usar las TIC en el aula es necesario previamente iniciar una profunda transformación sobre el rol que ocupa el docente. Para ello, se requiere un cambio de paradigma y actitudes, tal como exponen Casillas et al. (2020) y Gómez-Trigueros et al. (2019). Ya que el hecho de introducir tecnologías nos obliga a reformular la metodología implementada en el aula si queremos que esta sea eficiente y eficaz.

De la investigación de Konca et al. (2016), se concluye la necesidad de dotar de conocimientos a los docentes para que no solo sean capaces de crear (rol prosumidor) o consumir material (rol consumidor), sino 
que desarrollen la capacidad para aprender a enseñar mediante las TIC. Ya no basta con sustituir el soporte impreso por otro digitalizado, se necesita ofertar mayor formación docente en competencias tecnológicas y usos pedagógicos (Gallardo et al., 2021).

Ante la situación actual, los docentes se han visto en la necesidad de adaptar sus saberes a las nuevas exigencias que les impone la cultura digital, especialmente durante la reciente crisis sanitaria a causa del Covid-19. Este hecho ha acelerado la digitalización de la educación y paradójicamente ha contribuido al incremento de la brecha digital por causas sociales (Portillo et al., 2020).

Esta necesidad de respuesta social se ha traducido, como veníamos avanzando, en una transformación de la figura del docente. En palabras de Moreno y Torres (2014) ese cambio como orientador y mediador tiene que pasar por guiar al alumnado y brindarles la oportunidad de aprovechar la información y la comunicación que nos ofrecen las TIC de manera didáctica, racional, crítica y analítica, pero siempre articulando los procesos con la realidad social y cultural. Las tecnologías son una oportunidad para lograr cambios profundos si contribuyen a mejorar los procesos de enseñanza aprendizaje desde nuevos planteamientos pedagógicos y curriculares (Gallardo et al., 2020).

Formar al alumnado en TIC es imprescindible, precisamente para que tengan un uso responsable y consciente del contenido que consumen. El objetivo perseguido debería ser que el conjunto del alumnado, después de un proceso de escolarización obligatoria, lleguen a ser ciudadanos competentes digitales en esta sociedad de la tecnología y la comunicación. Esta necesidad se materializa en el estudio publicado por Casillas et al. (2020) donde afirman que la actitud del alumnado hacia las TIC es favorable, pero su manejo y conocimiento es escaso para ser considerados nativos digitales.

En definitiva, la única vía para compensar esta carencia es la formación, en especial la formación del profesorado en Competencia Digital Docente con el fin de poder formar al alumnado en este campo (Del Pino, 2016; Portillo et al., 2020). Apostando tal como indica Domingo-Coscollola et al. (2020) por el desarrollo profesional del profesorado y la alfabetización digital a todos los niveles, especialmente de aquellos que serán futuros maestros y maestras (Girón et al. 2019). La competencia digital resulta cada vez más necesaria para poder participar de forma significativa en la nueva sociedad y economía del conocimiento del siglo XXI (INTEF, 2017).

\section{DISCUSIÓN Y CONCLUSIONES}

Llegado este momento, sintetizamos los principales hallazgos de esta investigación con respecto al objeto de estudio. Los resultados que surgen de la búsqueda son numerosos y la temática es amplia y compleja. La relación entre TIC y el proceso educativo es innegable y constituye un punto relevante en el universo de estudios realizados.

Es preciso señalar que una de las temáticas más relevantes en el contexto en que se realiza la búsqueda ha sido el uso de las TIC como herramienta para atender necesidades específicas e intervenir en dificultades de aprendizaje concretas. Debido a su alta especificidad, han sido mencionadas, pero no hemos profundizado en sus enfoques y contenidos.

Retomando las preguntas iniciales, pasamos a sintetizar el contenido de las investigaciones revisadas.

Respecto a las características de la infancia, las investigaciones parten de un enfoque pedagógico de la infancia como un momento clave en el desarrollo de las personas, que convendría incorporar y dar voz en el espacio público y político de nuestras sociedades. La preocupación por esta etapa educativa es, cada vez más, 
un asunto de interés comunitario y se tiende a favorecer mecanismos que garanticen el bienestar del menor, así como luchar contra la desigualdad que afecta en el proceso de inclusión socioeducativa. Las investigaciones se orientan hacia el estudio del cerebro del infante en relación al contexto y factores que le influyen durante los primeros años de vida con el fin de analizar en qué grado afecta a su capacidad de aprendizaje.

En cuanto a la relación entre infancia y tecnología, se concluye que la infancia se desarrolla, actualmente, en un contexto tecnológico que ofrece al niño/niña una serie variada de estrategias para conectarse y hacer uso de lo digital. Destaca el reducido número de investigaciones que se centran en analizar los efectos de las TIC en el proceso educativo sin focalizarse en experiencias concretas. En relación al contexto educativo, existen dos posiciones generalizadas: la de los autores y profesionales que consideran que la relación entre infantes y tecnología es beneficiosa para el proceso de enseñanza aprendizaje y desarrollo, y la de aquellos que rechazan las tecnologías alegando las consecuencias negativas que estas provocan en el alumnado. Asimismo, se evidencia una necesaria formación crítica y reflexiva sobre el uso de las TIC a los y las menores, así como en relación a los medios audiovisuales y su contenido. Las investigaciones que se centran en el uso de determinados recursos y procesos son variadas y profundizan en alto grado en la relación entre alumnado, objetivos de aprendizaje y tecnologías.

Respecto a la relación entre maestros y maestras y tecnología, resulta significativa la relación entre TIC y posibilidades de aprendizaje.No obstante, esta se ve limitada por la actitud y formación del docente ante el uso de la tecnología. Las evidencias encontradas indican que se está produciendo un cambio de paradigma hacia una combinación entre los planteamientos tecnológicos y un desarrollo coherente, crítico y solidario de los y las menores. Destaca el cambio en el rol del docente, el cual se provoca desde una posición, habitualmente, consumidora de recursos digitales hacia una posición prosumidora, creadora de contenidos. Al igual que en el caso del alumnado, resulta prioritario potenciar mecanismos de formación docente que respondan ante las necesidades del contexto específico en que se encuentran. En definitiva, que beneficien al desarrollo de la Competencia Digital Docente.

Concluimos destacando la relevancia de la formación inicial del profesorado, así como de su actitud ante el proceso educativo, como factores determinantes en los resultados que la incorporación de las TIC provoca en el proceso de enseñanza-aprendizaje del alumnado de Infantil. Pese a que el compromiso y la responsabilidad de los docentes está siendo muy relevante, Viñals y Cuenca (2016) consideran que, si la forma de aprender ha cambiado, también debe hacerlo la forma de enseñar del profesorado caminando hacia una escuela de transformación social en un mundo cambiante.

Asimismo, se señala la necesidad de acceder a las opiniones de las familias para conocer el rol del infante en el hogar, así como el del adulto en la relación TIC-infancia. Los datos evidencian una necesaria formación crítica y reflexiva sobre el uso de las tecnologías. Como trabajos futuros nos planteamos dos ámbitos: a) analizar en profundidad la relación entre escuela, familia y TIC y b) repensar la formación inicial y permanente del profesorado para poder educar en la escuela Infantil desde la igualdad, la equidad y la inclusión social. Este estudio sugiere nuevas preguntas de investigación:

¿En qué aspectos hemos de repensar tanto los enunciados como las prácticas referidas a la infancia o a las infancias, en los planos educativo, familiar, jurídico, político y cultural?

¿Cómo gestionar las propias exclusiones que el uso de las tecnologías está provocando en la escuela Infantil del siglo XXI?

¿Cómo potenciar una escuela infantil para la comprensión y la creación de cultura? 


\section{APOYOS}

Este artículo ha sido realizado mientras estamos inmersos en el Proyecto: Los materiales didácticos digitales en la Educación Infantil.Análisis y propuestas para su uso en la escuela y el hogar (INFANCI@DIGIT@L) en una muestra de tres comunidades autónomas (Galicia, Canarias y Valencia). IP 1: J. Rodríguez. Rodríguez. IP 2: M. Area Moreira.

\section{REFERENCIAS}

Andersen, H., \& Sorensen, E. (2017). Enhancing Understanding, Flow and Self-Efficacy in Learners with Developmental and Attention Difficulties through ICT-Based Interventions. European Journal of Open, Distance and E-Learning, 20(1), 153174. https://doi.org/10.1515/eurodl-2017-0010

Bauman, Z. (2020). Sobre la educación en un mundo líquido. Paidós Ibérica.

Bentley, D. (2018). "An idea is in your body": technology and transformation in the early childhood classroom. Global Studies of Childhood, 8(2), 162-172. https://doi. org/10.1177/2043610618760099

Camargo, D., \& Orozco, L. (2013). Factores asociados a la disponibilidad y uso de medios electrónicos en niños desde preesco lar hasta $4^{\circ}$ grado. Biomédica, 33, 175-185.

Cano-Díaz, L., Pulido-Álvarez, A., \& Giraldo-Huertas, J. (2015). Una mirada a la caracterización de la primera infancia: contextos y métodos. Revista Latinoamericana de Ciencias Sociales, $\mathrm{Ni}$ ñez Y Juventud, 13(1), 279-293. https://doi.org/10.11600/169 $\underline{2715 \times .13116250314}$

Cánovas, G. (2014). Menores de edad y conectividad móvil en España: tablets y smartphones. PROTÉGELES. https://bit. ly/3dKH8Tg

Cantillo, C. (2015). Imágenes infantiles que construyen identidades adultas. Los estereotipos sexistas de las princesas Disney desde una perspectiva de género. Efectos a través de las generaciones y en diferentes entornos: digital y analógico [Tesis Doctoral], Universidad Nacional de Educación a Distancia, España.

Casillas, S., Cabezas, M., \& García, F.J. (2020). Digital Competence of Early Childhood Education Teachers: Attitude, Knowledge and Use of ICT. European Journal of Teacher Education, 43(2), 210-223. https://doi.org/10.1080/02619768.2019.1681393

Correa, P., Carbia, P., Crestar, I., De Coo, M., Diz, M., \& Fernández, T. (2015). Propuesta de prevención primaria de la anorexia y bulimia en la infancia. Revista de Estudios e Investigación en Psicología y Educación, 5, 74-78. https://doi.org/10.17979/ reipe.2015.0.05.259

Del Pino, R. (2016). La competencia digital del profesorado de infantil y primaria en Canarias [Tesis Doctoral], Universidad de Las Palmas de Gran Canaria, Las Palmas de Gran Canaria, España.

Delors, J. (1996). La educación encierra un tesoro: Informe a la Unesco de la Comisión Internacional sobre la educación para el siglo XXI. Santillana.

Díaz, R., \& Aladro, M. (2016). Relación entre uso de las nuevas tecnologías y sobrepeso infantil, como problema de salud pública. RqR Enfermería Comunitaria, 4(1), 46-51. http://bit. ly/3aj6zcx

Domingo-Coscolla, M., Bosco, A., Carrasco Segovia, S., \& Sánchez Valero, J.A. (2020) Fomentando la competencia digital docente en la universidad: Percepción de estudiantes y docentes. Revista de Investigación Educativa, 38(1), 167-782. http:// dx.doi.org/10.6018/rie.340551

Dubinsky, J. M., Guzey, S. S., Schwartz, M. S., Roehrig, G., MacNabb, C., Schmied, A. Hinesley, V., Hoelscher, M., Michlin, M., Schmitt, L., Ellingson, C., Chang, Z., \& Cooper, J. L. (2019). Contributions of neuroscience knowledge to teachers and their practice. The Neuroscientist, 25(5), 394-407. https://doi. org/10.1177/1073858419835447

Espigares-Gámez, M.J., Fernández-Oliveras, A., \& Oliveras, M.L. (2020). Juegos como potenciadores de aprendizajes STEAM. 
Aplicación de juegos tradicionales jamaicanos en Educación Intercultural Infantil y Primaria. Acta Scientaiae, 22(4), 28-50. https://doi.org/10.17648/acta.scientiae.6019

Espino-Díaz, L., Fernández-Caminero, G., Hernandez-Lloret, C.M., Gonzalez-Gonzalez, H. \& Alvarez-Castillo, J.L. (2020). Analyzing the impact of COVID-19 on education professionals. toward a paradigm shift: ICT and neuroeducation as a binomial of action. Sustainability, 12(14), e5646. https://doi. org/10.3390/su12145646

European Commission (2015). Young children (0-8) and digital technology: a qualitative exploratory study across seven countries. Publicaciones de la Unión Europea.

Fernández, J.M. (2013). Competencias docentes y educación inclusiva. Revista Electrónica de Investigación Educativa, 15(2), 82-99. http://bit.ly/3ajEwtg

Gallardo, I.M., Castro, A., \& Saiz, H. (2020). Interacción y uso de Tecnologías en los procesos de enseñanza y aprendizaje. Educatio Siglo XXI, 38(1) 119-138. https://doi.org/10.6018/ educatio. 413441

Gallardo, I.M., Mariño, R., \& Vega, A. (2021). Creación de materiales didácticos digitales y uso de tecnologías por parte de los docentes de Primaria. Un estudio de casos. Revista Ibero-americana de Educación, 85(1) 39-60. https://doi. org/10.35362/rie8514063

García, I., \& P. Gutiérrez, P. (2020). El rol docente en la sociedad digital. Digital Education Review, (38), 1-22. https://doi. org/10.1344/der.2020.38.1-22

García-Perales, R., \& Almeida, L. (2019). An Enrichment Program for Students with High Intellectual Ability: Positive Effects on School Adaptation. Comunicar: Media Education Research Journal, 27(60), 39-47. https://doi.org/10.3916/C60-2019-04

Gimeno, J. (2013). El currículum: una reflexión sobre la práctica. Morata.

Girón, V., Cózar, R., \& González-Calero, J.A. (2019). Análisis de la autopercepción sobre el nivel de competencia digital docente en la formación inicial de maestros/as. Revista Electrónica Interuniversitaria de Formación del Profesorado, 22(3), 193218. https://doi.org/10.6018/reifop.373421
Gómez, M. (2016). La pizarra digital como recurso innovador y favorecedor del proceso de enseñanza/aprendizaje de los alumnos de Infantil y Primaria del municipio de Alcorcón, Madrid [Tesis Doctoral]. Universidad Nacional de Educación a Distancia, España.

Gómez-Trigueros, I.M., Ruiz-Bañuls, M., \& Ortega-Sánchez, D. (2019). Digital Literacy of Teachers in Training: Moving from ICTs (Information and Communication Technologies) to LKTs (Learning and Knowledge Technologies). Education Sciences, 9(4), e274. https://doi.org/10.3390/educsci9040274

González, E. (Coord.) (2007). Introducción temprana a las TIC: estrategias para educar en un uso responsable en Educación Infantil y Primaria. Secretaría General Técnica. https://bit. ly/3pCvxZr

González, M. (2019). Niño nace. Revista GEARTE, 6(2), 168-178. http://bit.ly/2LaGUcg

Grindheim, L. (2017). Children as Playing Citizens. European Early Childhood Education Research Journal, 25(4), 624-636. https://doi.org/10.1080/1350293X.2017.1331076

INTEF (2017). Marco Común de Competencia Digital. https://goo. gl/ R9ia89

Kerckaert, S., Vanderlinde, R., \& van Braak, J. (2015). The role of ICT in early childhood education: scale development and research on ICT use and influencing factors. European Early Childhood Education Research Journal, 23(2), 183-199. https://doi.org/10.1080/1350293X.2015.1016804

Konca, A., \& Koksalan, B. (2017). Preschool children's interaction with ICT al home. International Journal of Research in Education a Science, 3(2), 571-581.http://bit.ly/3rlioAr

Konca, A., Ozel, E. \& Zelyurt, H. (2016). Attitudes of Preschool Teachers towards Using Information and Communication Technologies (ICT). International Journal of Research in Education and Science, 2(1), 10-15. http://bit.ly/37aLhLX

Leo, M.J., Morera, M. \& Rodríguez, M. (2018). Las TIC dan la mano a la Educación Infantil. Hachetetepé: Revista científica de Educación y Comunicación, 16, 107-120. https://doi.org/10.25267/ Hachetetepe.2018.v1.i16.10 
Lindahl, M.G., \& Folkesson, A. (2012). ICT in Preschool: friend or foe? The significance of norms in a changing practice. International Journal of Early Years Education, 20(4), 422-436. https://doi.org/10.1080/09669760.2012.743876

Moreno, C.H. \& Torres, L.M. (2014). Estado del arte sobre las TIC desde los programas de formación de maestros de educación infantil [Tesis de grado]. Universidad De San Buenaventura, Bogotá, Colombia.

Marcino, P. (2018). Impact of Information and Communication Technology on Academic Achievement for Exceptional Student Education Inclusion Students [Tesis Doctoral]. Universidad Walden, Minnesota, Estados Unidos.

Marcos, L., \& Ubrich, T. (2017). Desheredados: desigualdad infantil, igualdad de oportunidades y políticas públicas en España. Save the Children. https://goo.gl/mfka3d

Marqués, P. (2014). Metainvestigación 2013-14. Uso educativo de las tabletas digitales en Educación Infantil. Informe Final. http://bit.ly/3oZ8GX1

Masoumi D. (2015). Preschool teachers' use of ICTs: Towards a typology of practice. Contemporary Issues in Early Childhood, 16(1), 5-17. https://doi.org/10.1177/1463949114566753

Mohaned, A. (2018). Teachers' Perspectives Surrounding ICT Use amongst SEN Students in the Mainstream Educational Setting. World Journal of Education, 8(1), 6-16. https://doi. org/10.5430/wje.v8n1p6

Mølster, T. (2018). To What Extent Does Information and Communication Technology Support Inclusion in Education of Students with Learning Difficulties? Universal Journal of Educational Research, 6(4), 598-612. https://doi.org/10.13189/ ujer.2018.060403

Molina, N. (2005). Herramientas para investigar ¿Qué es el estado del arte? Ciencia y Tecnología para la salud Visual y Ocular, 5 , 73-75. http://bit.ly/32y7qzw

Morin, E. (2020). Cambiemos de vía. Lecciones de la pandemia. Paidós.

Muñoz, O. (2014). La era de la niñez digital: la antítesis como propuesta. Rayuela, 10, 246-251. https://bit.ly/2LLffyl

Murillo, F., \& Martínez, C. (2018). Magnitud de la segregación escolar por nivel socioeconómico en España y sus Comunida- des Autónomas y comparación con los países de la Unión Europea. Revista de Sociología de la Educación, 11(1), 37-58. https://doi.org/10.7203/RASE.11.1.10129

Navarro, N. (2014). Los mensajes televisivos y la infancia [Tesis Doctoral]. Universidad Complutense de Madrid, Madrid, España.

Newman, N. (2018). Perspectives: early childhood, media use, and development-human touch first and foremost. Zero to three, 39(2), 36-38.

Norehan, H., Hamizah, N., Mohammad, R., Ahmad, N., Habibah, H., Zaki, M., Mohamed, R., \& Shamsul, S. (2017). Grooming Future Scientists and Engineers from the Root through Fun Learning Concept. Journal of Physics: Conf. Series, 180, 1-11. https://doi.org/10.1088/1742-6596/1174/1/012003

PACER Center (2014). Assistive technology for infants, toddlers, and young children with disabilities. http://bit.ly/3pY1kEz

Palacio, C., Tobón, J., Toro, D., \& Vicuña, J. (2018). El Estrés escolar en la Infancia: Una reflexión Teórica. Panamerican Journal of Neuropsychology, 12(2), 1-16. https://doi.org/10.7714/ CNPS/12.2.206

Perrin, A.L. (2019). Raising teachers' awareness of cognitive sciences and neuroeducation: effects on professional representations and actions. Recherche Formation, 2, 89-104. https:// doi.org/10.4000/rechercheformation.5532

Pinto, M., \& Osório, A.J. (2019). Aprender a programar en Educación Infantil: Análisis con la escala de participación. PixelBit. Revista de Medios y Educación, (55), 133-156. https://doi. org/10.12795/pixelbit.2019.i55.08

Pombo, A. (2019). El duelo en la Infancia. Intercambios: Papeles de Psicoanálisis, 42, 75-83. https://bit.ly/36qzMQe

Portillo, J., Garay, U., Tejada, E., \& Bilbao, N. (2020). Self-Perception of the Digital Competence of Educators during the COVID-19 Pandemic: A Cross-Analysis of Different Educational Stages. Sustainability, 12(23), e10128. https://doi. org/10.3390/su122310128

Quintana, E. (2019). Strong Families Thriving Children. Morrison Institute for Public Policy. https://bit.ly/3qltsSc

Ramada, L. (2017). Esto no va de libros. Literatura infantil y juvenil digital y educación literaria [Tesis Doctoral]. Universitat Autònoma de Barcelona, Barcelona, España. 
Ribeiro, M. (2019). Estabilidad diagnóstica y características del trastorno bipolar de inicio en la infancia y adolescencia en una muestra europea [Tesis Doctoral]. Universidad de Navarra, Pamplona, España.

Saiz, H. (2019). Los modelos didácticos y organizativos como factores determinantes en las transiciones de Educación Primaria a Educación Secundaria [Tesis doctoral]. Universitat de Valencia, Valencia, España.

Sánchez, L., Benítez, R., \& Aguilar, S. (2018). El triángulo de la educación infantil: los cuentos, las emociones y las TIC. Hachetetepé: Revista Científica de Educación y Comunicación, 16, 29-38. https://doi.org/10.25267/Hachetetepe.2018.v1.i16.4

Save the Children (2017). Memoria anual 2017. https://goo. $\mathrm{gl} / 7 \mathrm{doU} 7 \mathrm{~B}$

Siraj-Blatchford, J., \& Romero, R. (2017). De la aplicación a la participación activa de las TIC en Educación Infantil. PixelBit: Revista de Medios y Educación, (51), 165-181. https://doi. org/10.12795/pixelbit.2017.i51.11

Tagore, R. (1917). Personality. The Macmillan Company

UNESCO (2009). Directrices sobre políticas de inclusión en la educación. Unesco.

UNESCO (2015). Declaración de Incheon. Educación 2030. Unesco.

UNICEF (2018). Plan Estratégico 2018-2021. División de Comunicaciones de Unicef.

Vargas, G., \& Calvo, G. (1987). Seis modelos alternativos de investigación documental para el desarrollo de la práctica universitaria en educación. Educación Superior y Desarrollo, 5(3), 7-37. https://goo.gl/THb98A

Viñals A. \& Cuenca, J. (2016). El rol del docente en la era digital. Revista Interuniversitaria de Formación del Profesorado, 30(2), 103-114.

Wamuyu, P. (2017). Closing the Digital Divide in Low-Income Urban Communities: A Domestication Approach. Interdisciplinary Journal of e-Skills and Lifelong Learning, 13, 117-142. https://doi.org/10.28945/3885

Zabalza, M. (2018). Pedagogía(s) de infancia. RELAdEI. Revista Latinoamericana de Educación Infantil, 2(3), 17-36. http://bit. ly/39wM1N4 